\title{
Hidden Symmetries of Complex Analysis
}

\author{
Cao-Huu Tuan', Dorin Ghisa1, Allan Florin Muscutar ${ }^{2}$ \\ ${ }^{1}$ Glendon College, York University, Toronto, Canada \\ ${ }^{2}$ Department of Science and Mathematics, Lorain CCC, Elyria, OH, USA \\ Email: tuan@glendon.yorku.ca,dghisa@yorku.ca, fmuscuta@lorainccc.edu
}

How to cite this paper: Tuan, C.-H., Ghisa, D. and Muscutar, A.F. (2019) Hidden Symmetries of Complex Analysis. Advances in Pure Mathematics, 9, 844-856. https://doi.org/10.4236/apm.2019.910041

Received: April 26, 2019

Accepted: October 12, 2019

Published: October 15, 2019

Copyright $\odot 2019$ by author(s) and Scientific Research Publishing Inc. This work is licensed under the Creative Commons Attribution International License (CC BY 4.0).

http://creativecommons.org/licenses/by/4.0/

\begin{abstract}
We are dealing with domains of the complex plane which are not symmetric in common sense, but support fixed point free antianalytic involutions. They are fundamental domains of different classes of analytic functions and the respective involutions are obtained by composing their canonical projections onto the complex plane with the simplest antianalytic involution of the Riemann sphere. What we obtain are hidden symmetries of the complex plane. The list given here of these domains is far from exhaustive.
\end{abstract}

\section{Keywords}

Hidden Symmetry, Fundamental Domain, Antianalytic Involution

\section{Introduction}

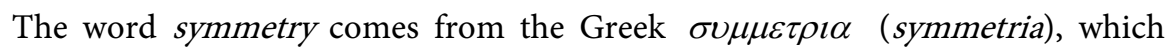
means "agreement in dimensions, due proportion, arrangement". It has an obvious visual connotation and it refers to a sense of harmonious and beautiful proportion and balance. It is highly conveyed in arts and architecture and appears frequently in mathematics, physics, biology and chemistry. In mathematics it refers to objects which are (after Felix Klein) invariant to some transformations, including reflections, rotations and scaling. The symmetry of the most of these objects is manifest. However, in complex analysis objects remaining invariant to some very simple groups of transformations are legitimately called symmetric, without appearing necessarily visually symmetric. These are the hidden symmetries of complex analysis. Some of them are instrumental in the construction of the family of Klein surfaces, which is a category including that of Riemann surfaces. These last surfaces are (see [1], p. 112) "domains of the most general type which can be used to replace the complex plane in the theory of analytic functions of one complex variable". A (bordered) Riemann surface $S$ is a 
connected Hausdorff space with a conformal structure defined by a family $\Phi$ of local homeomorphisms (parameters). Each $\varphi \in \Phi$ is a topological mapping of an open set $V \subset S$ onto a relatively open set of the upper (closed) half plane $\operatorname{Im} z \geq 0$ such that: if $V^{\prime} \subset V$, then $\varphi_{V^{\prime \prime}} \in \Phi$, if $V \subset S$ is covered by open sets $V^{\prime} \subset S$ such that $\varphi_{\mid V^{\prime \prime}} \in \Phi$, then $\varphi \in \Phi$ and finally the domains of all $\varphi$ cover the whole space $S$. The conformal structure of $\Phi$ means that for every $\varphi_{1}, \varphi_{2} \in \Phi, \varphi_{1} \circ \varphi_{2}^{-1}$, where defined, is a conformal mapping. When

$\varphi(V) \subset\{z \mid \operatorname{Im} z>0\}$ for every $\varphi \in \Phi$ we say that $S$ is a Riemann surface. Otherwise we denote $\beta(S)=\{s \in S \mid \operatorname{Im} \varphi(s)=0$ for some $\varphi \in \Phi\}$ and we call it the border of $S$ and $S$ is called bordered Riemann surface.

A symmetric Riemann surface $S$ is a (bordered, or border free) Riemann surface endowed with a fixed point free antianalytic involution $k$. This means that for every $\varphi_{1}, \varphi_{2} \in \Phi, \varphi_{2} \circ k \circ \varphi_{1}^{-1}$ is a mapping of $\varphi_{1}\left(V_{1}\right)$ onto $\varphi_{2}\left(V_{2}\right)$ whose complex conjugate is conformal. The involution $k$ generates the two-element group of transformations of $S$ formed with $k$ and the identity $I$. We have $k \circ I=I \circ k=k$ and $k \circ k=I$. This group is denoted by $\langle k\rangle$ and the respective symmetric Riemann surface is denoted by $(S, k)$. This is a symmetry in the sense of Klein (see [2]) and in this paper we will be talking only about this kind of symmetries. It is known that the conformal structure of $(S, k)$ induces a $d i$ analytic structure $\Psi$ on the factor space $X=S /\langle k\rangle$, i.e. local parameters $\psi \in \Psi$ are defined on $X$ by $\psi(\tilde{s})=\varphi(s)$, where $\tilde{s}=(s, k(s))$ such that for every $\psi_{1}, \psi_{2} \in \Psi$, we have that $\psi_{1} \circ \psi_{2}^{-1}$ is a conformal mapping or the conjugate of a conformal mapping, named anticonformal. The surface $(X, \Psi)$ obtained in this way is called Klein surface. Klein surfaces can be orientable when all $\psi_{1} \circ \psi_{2}^{-1}$ are conformal mappings or non orientable, when some of them are anticonformal. When $\beta(X) \neq \varnothing$ we have a bordered Klein surface and when $\beta(X) \neq \varnothing$ we have a border free Klein surface.

\section{Simple Examples of Hidden Symmetries}

The simplest examples of symmetric Riemann surfaces are the complex plane and the Riemann sphere endowed with the fixed point free antianalytic involution $k(z)=-1 / \bar{z}$. The Klein surfaces generated by these symmetric Riemann surfaces are the non orientable real projective plan, respectively the Klein bottle, which is also a non orientable surface. When the symmetric bordered Riemann surface is the annulus $A_{R}, 1 / R \leq|z| \leq R$ endowed with the induced analytic structure of the complex plane, the generated non orientable Klein surface is the Möbius strip $M_{R}$. Figure 1 illustrates the kind of symmetry $k(z)$ is representing and how the Möbius strip is obtained by this symmetry. This picture has been created by Ove Edfors at the request of Ilie Barza. Here the symmetry is obvious. The image of the disc

$$
\left|z-R \mathrm{e}^{i \theta_{0}}\right| \leq r, r<R
$$

by $k(z)$ is the disc 


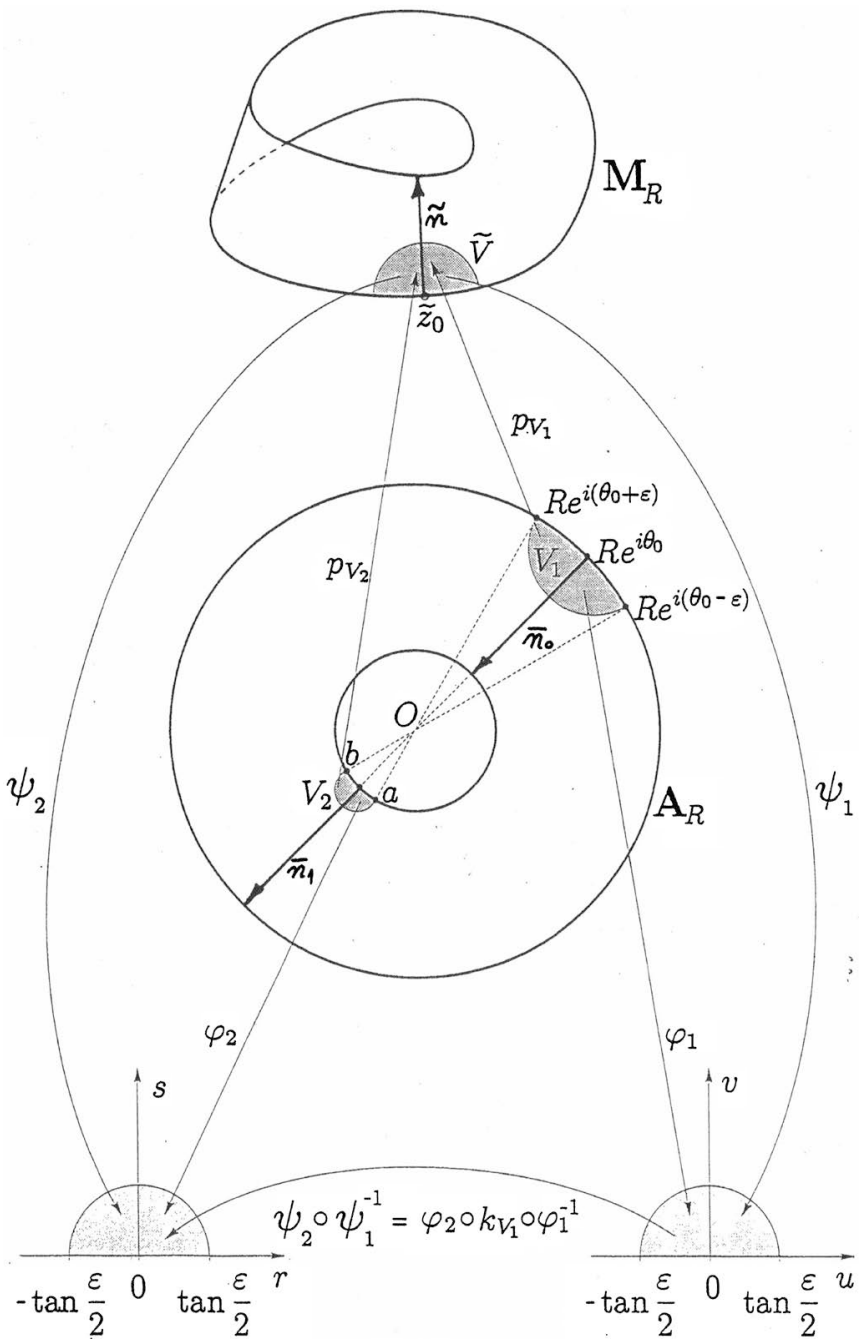

Figure 1 . The symmetry defined by $k(z)=-1 / \bar{z}$ and the construction of the Möbius strip as a non orientable Klein surface.

$$
\left|z-z_{0}\right| \leq \frac{r}{R^{2}-r^{2}}, \text { where } z_{0}=\frac{r}{R^{2}-r^{2}} \mathrm{e}^{i\left(\theta_{0}+\pi\right)} .
$$

Local parameters $\varphi_{1}$ and $\varphi_{2}$ can be defined on $V_{1}=A_{R} \cap\left\{z|| z-R \mathrm{e}^{i \theta_{0}} \mid \leq r\right\}$, respectively $V_{2}=A_{R} \cap\left\{z|| z-z_{0} \mid \leq \frac{r}{R^{2}-r^{2}}\right\}$ by

$$
\varphi_{1}(z)=\frac{z-R \mathrm{e}^{i \theta_{0}}}{a z+b},
$$

where $a$ and $b$ are uniquely determined such that

$$
\varphi_{1}\left(R \mathrm{e}^{i\left(\theta_{0}+\epsilon\right)}\right)=\tan \frac{\epsilon}{2} \text { and } \varphi_{1}\left(R \mathrm{e}^{i\left(\theta_{0}-\epsilon\right)}\right)=-\tan \frac{\epsilon}{2} .
$$

Analogously

$$
\varphi_{2}(z)=\frac{z-(1 / R) \mathrm{e}^{i\left(\theta_{0}+\pi\right)}}{c z+d}
$$


where $c$ and $d$ are determined such that

$$
\varphi_{2}\left(\frac{1}{R} \mathrm{e}^{i\left(\theta_{0}+\epsilon+\pi\right)}\right)=-\tan \frac{\epsilon}{2} \text { and } \varphi_{2}\left(\frac{1}{R} \mathrm{e}^{i\left(\theta_{0}-\epsilon+\pi\right)}\right)=\tan \frac{\epsilon}{2} .
$$

If $p$ is the projection of $A_{R}$ onto $M_{R}$, i.e. for $\tilde{z} \in M_{R}$ we have $\tilde{z}=p(z)=p(k(z))$, then $\psi_{j}=p_{\mid V_{j}}^{-1} \circ \varphi_{j}, j=1,2$ are local parameters on $M_{R}$ at $\tilde{V}=p\left(V_{1}\right)=p\left(V_{2}\right)$.

We have proved in [3], Theorem 3 that conformal images of symmetric domains are symmetric. Since Möbius transformations

$$
w=\chi(z)=\frac{a z+b}{c z+d}, a d-b c \neq 0
$$

are conformal mappings of the Riemann sphere (where $\chi(-c / d)=\infty$ and $\chi(\infty)=a / c)$ and having in view this theorem, we are expecting that composed with $k(z)$ the Möbius transformations will generate symmetries. Indeed we can prove

Theorem 1. For every Möbius transformation $w=\chi(z)$ the function $h=\chi \circ k \circ \chi^{-1}$ is a fixed point free antianalytic involution of the Riemann sphere.

Proof: Indeed, it can be easily checked that

$$
\chi^{-1}(w)=\frac{b-d w}{c w-a}, \chi \circ k(z)=\frac{b \bar{z}-a}{d \bar{z}-c}
$$

and therefore

$$
h(w)=\chi \circ k \circ \chi^{-1}(w)=\frac{\mu-A \bar{w}}{\bar{A}-v \bar{w}},
$$

where $A=a \bar{c}+b \bar{d}, \mu=|a|^{2}+|b|^{2}$ and $v=|c|^{2}+|d|^{2}$.

For the function $h(w)$ defined on the Riemann sphere we have $h(\infty)=A / v$ and $h(A / v)=\infty$.

Obviously, $h$ is an involution and an anticonformal mapping of the Riemann sphere and by [3] Theorem 3, it has no fixed point. We can prove by direct computation that $h$ is fixed point free. Indeed, any Möbius transformation is obtained by composing some particular elementary transformations as translations, rotations, magnifications and inversions. It can be easily checked that composed with $k$ as in the theorem each one of them generates fixed point free antianalytic involutions of the Riemann sphere. Finally an easy computation shows that if $\varphi \circ \psi \circ k \circ \psi^{-1} \circ \varphi^{-1}$ has a fixed point, then necessarily $\varphi$ has a fixed point, hence the function $h$ from the theorem is a fixed point free antianalytic involution.

\section{Blaschke Product Symmetries}

A Blaschke factor is a Möbius transformation of the form

$$
b_{n}(z)=\mathrm{e}^{i \theta_{n}} \frac{a_{n}-z}{1-\bar{a}_{n} z}
$$

where $\left|a_{n}\right|<1$ and $\theta_{n} \in \mathbb{R}$ 
A Blaschke product is an expression of the form

$$
w=B(z)=\prod_{n=1}^{m \leq \infty} b_{n}(z)
$$

When $m$ is finite we have finite Blaschke products which are rational functions of degree $m$, and when $m$ is infinite $B(z)$ is a transcendental function. It is not defined on the set $E$ of cluster points of $A=\left\{1 / \bar{a}_{n}\right\}$. In the infinite case it is customary to assign the values $\bar{a}_{n} /\left|a_{n}\right|$ to the constants $\mathrm{e}^{i \theta_{n}}$, as long as $a_{n} \neq 0$. The points $1 / \bar{a}_{n}$ are poles of $B(z)$. It is known that in the infinite case a necessary and sufficient condition for the uniform convergence of $B(z)$ on compact subsets of $\mathbb{C} \backslash(A \cup E)$ is $\sum_{n=1}^{\infty}\left(1-\left|a_{n}\right|\right)<\infty \quad$ (the Blaschke condition). Thus, $B(z)$ has a meaning only if $E$ is a subset of the unit circle. For finite Blaschke products $E=\varnothing$. Since every Blaschke factor maps the unit circle onto itself, the same is true for any Blaschke product, with the specification that in the infinite case $E$ should be removed. Also, the unit disc is mapped onto itself and the exterior of the unit disc is mapped onto itself. In general, these mappings are not bijective, yet there is a way to partition the complex plane into sets whose interiors are conformally mapped onto the whole complex plane with some slits. These are the fundamental domains of the Blaschke product.

For the simple case of a single zero of order $m \geq 2$, not only the fundamental domains are obvious, but so are the involved symmetries. Let

$$
B_{a}(z)=\left[\frac{\bar{a}}{|a|} \frac{z-a}{1-\bar{a} z}\right]^{m}, a=r \mathrm{e}^{i \alpha}, 0 \leq r<1, \alpha \in \mathbb{R}
$$

We can find the fundamental domains of $B_{a}(z)$ by solving the equation $B_{a}(z)=\lambda^{m}$, for $0 \leq \lambda \leq 1$. We get the solutions

$$
z_{n}(\lambda)=\frac{\omega_{n} \lambda+r}{\omega_{n} \lambda r+1} \mathrm{e}^{i \alpha}, n=0,1, \cdots, n-1,
$$

where $\omega_{n}$ are the roots of order $m$ of the unity. As $\lambda$ varies from 0 to 1 the point $z_{n}(\lambda)$ describes an arc $\gamma_{n}$ joining $z=a$ with the point $\frac{\omega_{n}+r}{\omega_{n} r+1} \mathrm{e}^{i \alpha}$ on the unit circle. It can be easily checked that $z_{n}(\lambda)$ are distinct numbers for $\lambda \neq 0$ and then those points on the unit circle are also distinct. They determine $m$ arcs $\delta_{n}$ on the unit circle which are mapped bijectively by $B_{a}(z)$ onto the the whole unit circle. The arcs $\gamma_{n}+\delta_{n}-\gamma_{n+1}$ bound domains which are mapped conformally by $B_{a}(z)$ onto the unit disc with a slit alongside the interval $(0,1)$ of the real axis. The symmetric domains with respect to the unit circle are mapped conformally by $B_{a}(z)$ onto the exterior of the unit disc with a slit alongside the interval $(1,+\infty)$. The union of the symmetric domains and of their common boundary are fundamental domains $\Omega_{j}$ of $B_{a}(z)$ which are mapped conformally by this function onto the complex plane with a slit alongside the positive real half axis. The antianalytic involution $k(w)=-1 / \bar{w}$ maps the upper half plane onto the lower half plane and vice-versa and the positive real half axis onto the negative real half axis and vice versa. Since the positive 
real half axis is the border of $B_{a}\left(\Omega_{j}\right)$ we cannot compose $B_{a \mid \Omega_{j}}^{-1}$ with $k \circ B_{a}(z)$ for $z \in \partial \Omega_{j}$ therefore instead of $\Omega_{j}$ we should take $\Delta_{j}=\Omega_{j} \backslash B_{a \mid \Omega_{j}}^{-1}(\{w \mid \operatorname{Im} w=0\})$ and define for $z \in \Delta_{j}$ :

$$
h_{j}(z)=B_{a \mid \Delta_{j}}^{-1} \circ k \circ B_{a}(z) .
$$

We notice that $\Delta_{j}$ is also a fundamental domain of $B_{a}(z)$ and it is symmetric in the sense of Klein with respect to the two-element group $\left\langle h_{j}, I\right\rangle$ where $I$ is the identity. We say that $h_{j}$ is a hidden symmetry of $\Delta_{j}$. Thus we have proved

Theorem 2. For every Blaschke product $B_{a}(z)$ of the form (12) there is a partition of the complex plane into $m$ sets whose interior are fundamental domains of $B_{a}(z)$ and which contains each one of them a hidden symmetry.

Figure 2 (see [4], page 16) illustrates these symmetric domains when $m=6$ and $a=1 / 2+i / 3$.

Let us see what is the pre-image of the real axis in the case of an arbitrary finite Blaschke product. The pre-image of the origin is the set $\left\{a_{n}\right\}$ of the zeros of $B(z)$. When performing continuation over the positive real half axis Re $w>0$ starting from a zero $z=a_{n}$ we obtain a Jordan arc with the end on the unit circle corresponding to a certain root of the equation $w=B(z)=1$, then it continues with a symmetric arc with respect to the unit circle such that the pole $1 / \bar{a}_{n}$ is reached at the limit as $w \rightarrow+\infty$. Hence the pre-image of the positive real half axis is formed with arcs connecting zeros and poles of $B(z)$. Continuing over the negative half axis we might reach the branch points of $B(z)$ and this will allow us to find fundamental domains of $B(z)$. This is the case of Blaschke products which have symmetric zeros with respect to the origin. Figure 3 illustrates the case of the Blaschke product

$$
B(z)=\frac{z-a}{1-\bar{a} z} \frac{z+a}{1+\bar{a} z} \frac{z-\bar{a}}{1-a z} \frac{z+\bar{a}}{1+a z}
$$

The roots of the equation $B(z)=1$ are \pm 1 and $\pm i$ and $B(0)=|a|^{4}$,

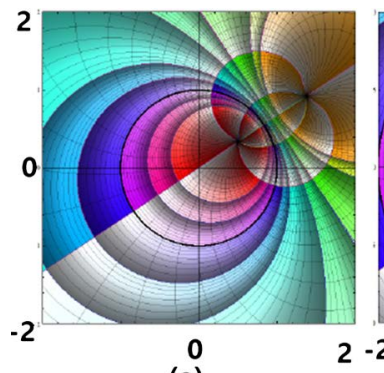

(a)

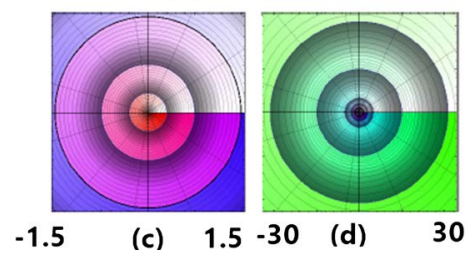

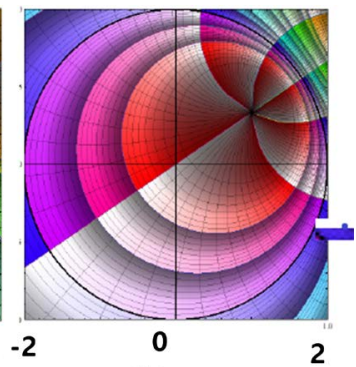

(b) 
$B(\infty)=1 /|a|^{4}$. Moreover, it can be easily checked that $\operatorname{Im} B(z)=0$ if $\operatorname{Im} z=0$, $B\left(\mathrm{e}^{k \pi i / 2} z\right)=B(z), k=0,1,2,3$ therefore the real and imaginary semi axes are mapped bijectively by $B(z)$ onto the interval $\left[|a|^{4}, 1 /|a|^{4}\right]$ and every quadrant is mapped conformally by $B(z)$ onto the complex plane with a slit alongside this interval. We notice that the antianalytic involution $k(z)=-1 / \bar{z}$ maps bijectively this interval onto the interval $\left[-1 /|a|^{4},-|a|^{4}\right]$. If we denote by $\Omega$ any one of these quadrants, and $s \in \Omega$ is such that $B(s) \in\left[-1 /|a|^{4},-|a|^{4}\right]$ then $k \circ B(s) \in\left[|a|^{4}, 1 /|a|^{4}\right]$ and $B_{\mid \Omega}^{-1} \circ k \circ B(s)$ is not defined at $s$. Then instead of $\Omega$ we should deal with $\Delta=\Omega \backslash B_{\mid \Omega}^{-1}\left(\left[-1 /|a|^{4},-|a|^{4}\right]\right)$ and study $h(s)=B_{\Delta}^{-1} \circ k \circ B(s)$. This is a hidden symmetry of $\Delta$. Figure 3 below illustrates this situation when $a=\frac{1}{2}(1+i)$. We colored blue the pre-image of the positive real half axis, red that of the negative real half axis, black that of the circle $|w|=1$, green that of the circle $|w|=0.5$ and brown that of the circle $|w|=1.5$.

Obviously, similar situations can be created when taking $a=r \mathrm{e}^{2 i \pi / m}, 0<r<1$ and dealing with the Blaschke product having the zeros $a_{k}=a \mathrm{e}^{\frac{2 k}{m} \pi i}, k=0,1, \cdots, m-1$. In the case where the zeros of $B(z)$ are randomly located inside the unit disc, we need to find a different approach.

Suppose $B(z)$ is a finite Blaschke product of degree $m$ :

$$
B(z)=\prod_{n=1}^{m} \frac{\bar{a}_{n}}{\left|a_{n}\right|} \frac{z-a_{n}}{1-\bar{a}_{n} z}
$$

Let us notice that

$$
B^{\prime}(z)=-B(z) \sum_{n=1}^{m} \frac{1-\left|a_{n}\right|^{2}}{\left(a_{n}-z\right)\left(1-\bar{a}_{n} z\right)}
$$

and for $|\zeta|=1$ we have

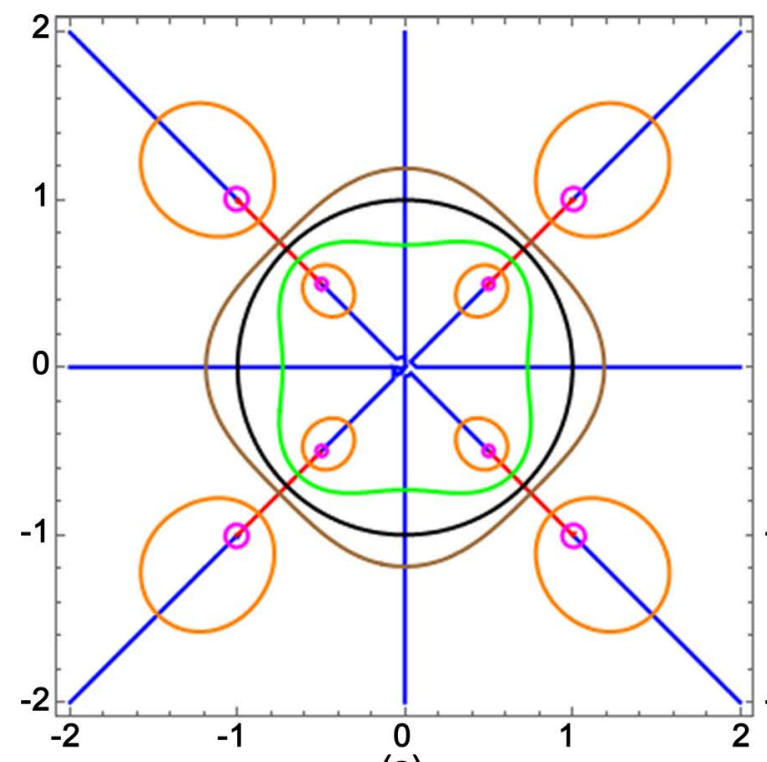

(a)

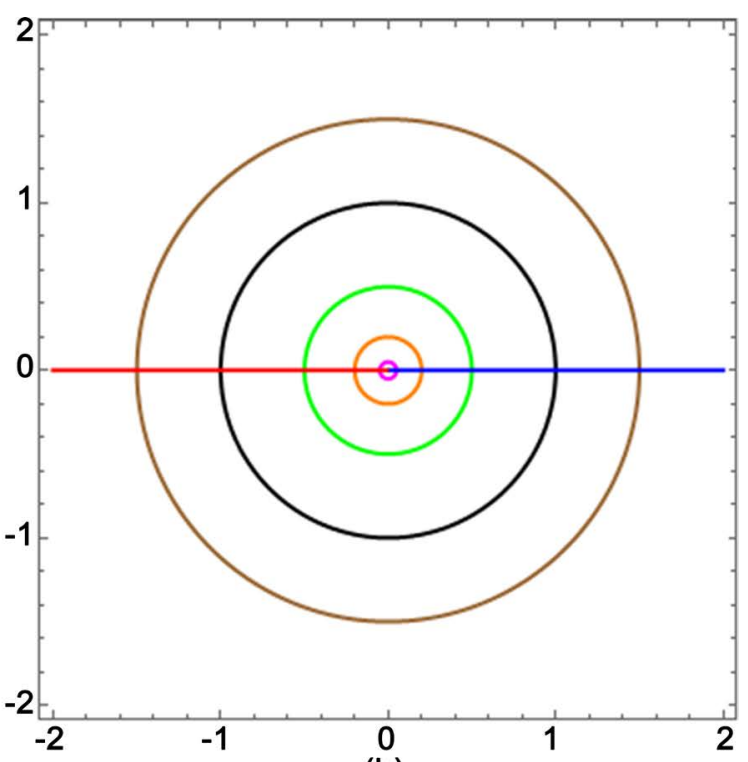

(b)

Figure 3. Fundamental domains of Blaschke products with symmetric zeros. 


$$
\left|B^{\prime}(\zeta)\right|=\sum_{n=1}^{m} \frac{1-\left|a_{n}\right|^{2}}{\left|a_{n}-\zeta\right|^{2}}>0
$$

therefore $B^{\prime}(z)$ does not cancel on the unit circle, which means that the roots of the equation $B(z)=\mathrm{e}^{i \alpha}, \alpha \in \mathbb{R}$, which are all on the unit circle, are simple roots no matter if the zeros of $B(z)$ are simple or not. On the other hand the equation $B^{\prime}(z)=0$ has $m-1$ roots (counted with multiplicities) inside the unit disc and they are instrumental in proving the following theorem (see [4], page 11):

Theorem 3. For any Blaschke product $B(z)$ of degree $m$ there is a partition of the complex plane into $m$ sets whose interior are fundamental domains of $w=B(z)$. These domains are symmetric with respect to the unit circle.

Proof: There is a constructive proof of this theorem (see [4], page 10). Namely, if $b_{j}, j=1,2, \cdots, m-1$ are the zeros of $B^{\prime}(z)$ inside the unit disc (counted with multiplicities), then $\left|B\left(b_{j}\right)\right|<1$ and there is a non self intersecting polygonal line $L$ connecting the points $B\left(b_{j}\right)$ and $w=1$. The pre-image of this line is formed with Jordan arcs $\gamma_{k}$ connecting the points $\zeta_{k}, k=1,2, \cdots, m$ on the unit circle, which are the solutions of the equation $B(z)=1$, with $b_{j}$. Some of these arcs can overlap, but they do not intersect each other in other points than $b_{j}$, since such an intersection point is a branch point of $B(z)$, i.e. a root of the equation $B^{\prime}(z)=0$. If $\eta_{k}$ are the arcs determined on the unit circle by consecutive points $\zeta_{k}$ and $\zeta_{k+1}$, then $\gamma_{k}+\eta_{k}+\gamma_{k+1}$ is a closed curve bounding a domain $D_{k}$ which is mapped conformally by $B(z)$ onto the open unit disc with a slit alongside the part of $L$ which is the image of $\gamma_{k}+\gamma_{k+1}$. The symmetric domain $\tilde{D}_{k}$ of $D_{k}$ with respect to the unit circle is mapped conformally by $B(z)$ onto the exterior of the unit disc with a slit alongside the image of the symmetric $\tilde{\gamma}_{k}+\tilde{\gamma}_{k+1}$ of $\gamma_{k}+\gamma_{k+1}$ with respect to the unit circle. The domains $\Omega_{k}=D_{k} \cup \tilde{D}_{k} \cup \eta_{k}$ (where $\eta_{k}$ are considered as point sets) are fundamental domains of $B(z)$ and they are mapped conformally by $B(z)$ onto the complex plane with a slit alongside the image of $\gamma_{k}+\tilde{\gamma}_{k}$ which is the same as that of $\tilde{\gamma}_{k+1}+\gamma_{k+1}$. It is obvious that they are symmetric with respect to the unit circle. Each one of them contains also the hidden symmetry $h_{j}(z)=B_{\mid \Omega_{j}} \circ k \circ B(z)$. This situation is illustrated in Figure 4.

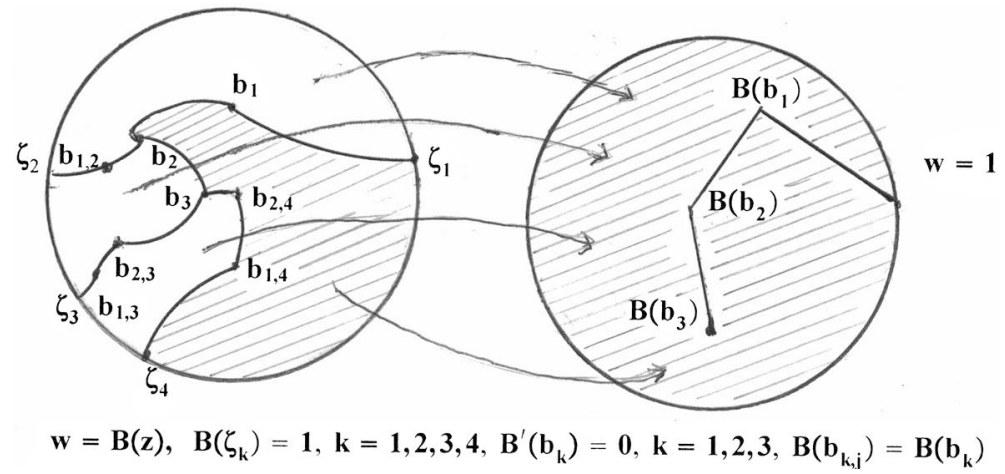

Figure 4. Building fundamental domains of Blaschke products with randomly chosen zeros. 


\section{The Case of Elementary Transcendental Functions}

We include in this family of functions the exponential $\mathrm{e}^{z}$, the trigonometric functions $\sin z, \cos z$ etc. as well as the hyperbolic functions $\sinh z, \cosh z$ etc. All of these functions are combinations of simple transformations of $\mathrm{e}^{z}$ and therefore it will be enough to deal with just one of them. In [5], page 99 as well as in [4], page 51 it is the function $\varphi(z)=\cos z$ which has been chosen. Figure 5 below illustrate the fundamental domains of this function which are vertical strips $\Omega_{j}$ bounded by the lines $\operatorname{Re} z=j \pi$ and $\operatorname{Re} z=(j+1) \pi, j \in \mathbb{Z}$. They are mapped conformally by $\cos z$ onto the complex plane with a slit alongside the real axis complementary to the interval $(-1,1)$. The antianalytic involution $k(z)=-1 / \bar{z}$ maps the upper half plane onto the lower half plane and vice-versa and then $\varphi_{\Omega_{j}}^{-1} \circ k \circ \varphi(z)$ is a hidden symmetry of $\Omega_{j}$. Figure 5 illustrates the fundamental domain of $\cos z$.

\section{The Modular Function $\lambda(\tau)$}

The modular function $\lambda(\tau)$ effects a one-to-one conformal mapping (see [5], page 281) of the domain $\Omega$ below (Figure 6) onto the upper half plane. The mapping extends continuously to the boundary in such a way that $\tau=0,1, \infty$ correspond to $\lambda=1, \infty, 0$. By Schwarz symmetry principle, the domain $\Omega^{\prime}$ symmetric to $\Omega$ with respect to the imaginary axis is mapped onto the lower half plane and then $\Omega \bigcup \Omega^{\prime} \bigcup\{\tau \mid \operatorname{Re} \tau=0, \operatorname{Im} \tau>0\}$ is mapped onto the whole complex plane with a slit alongside the real axis from $-\infty$ to 1 . This is a fundamental domain of $\lambda(\tau)$. If instead $\Omega^{\prime}$ we take $\Omega^{\prime \prime}$ the symmetric of $\Omega$

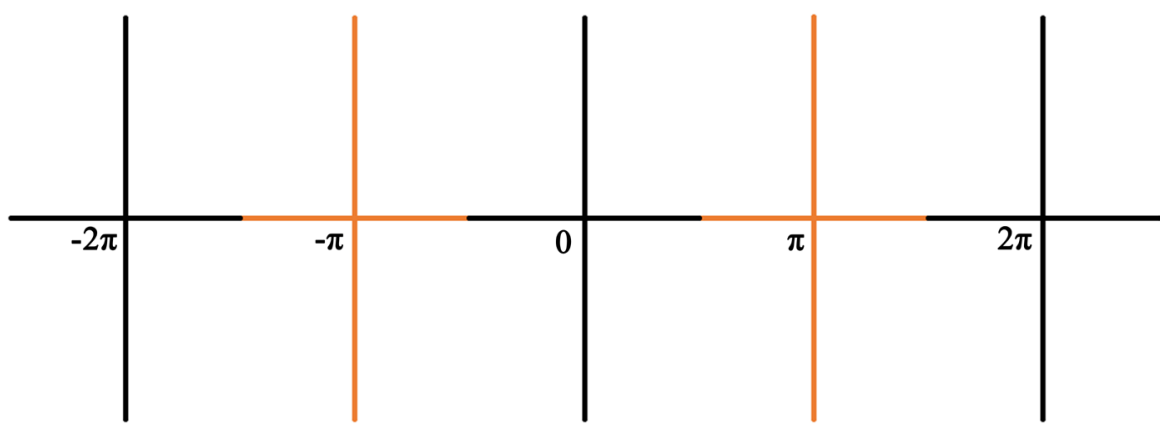

Figure 5. Fundamental domains of the function $\cos z$.

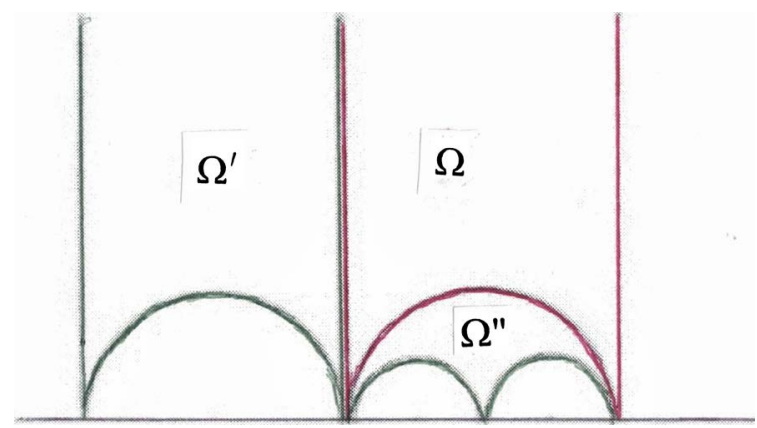

Figure 6. Fundamental domains of the modular function. 
with respect to the circle $|\tau-1 / 2|=1 / 2$ and we do the corresponding union we obtain another fundamental domain and the process can be continued indefinitely. The symmetric of these domains with respect to the real axis are also fundamental domains of $\lambda(\tau)$. These domains accumulate to every point of the real axis in the sense that any neighborhood of such a point contains infinitely many fundamental domains. There are obvious symmetries of these domains, yet each one of them, say $\Delta$ contains also a hidden symmetry, namely $\lambda_{\Delta}^{-1} \circ k \circ \lambda(\tau)$.

\section{The Hidden Symmetries of the Euler Gamma Function}

There are a lot of ways to introduce this famous function. For our purpose the Weierstrass definition is the most useful, namely:

$$
\Gamma(z)=\frac{\mathrm{e}^{-\gamma z}}{z} \prod_{n=1}^{\infty}\left(1+\frac{z}{n}\right)^{-1} \mathrm{e}^{z / n}
$$

where $\gamma=0.57722 \cdots$ is the Euler constant.

It is obvious from this formula that $\Gamma(z)$ is a meromorphic function in the complex plane having the simple poles $0,-1,-2, \cdots$ and no zero. As shown in [6] the pre-image by $\Gamma(z)$ of the real axis is formed with the infinitely many unbounded curves (components). The components corresponding to the negative and the positive real half axis alternate and do not cross each other. However, at every pole of $\Gamma(z)$ there are two such different components that meet each other. There are two types of components: those originating in the first and the last quadrant, which have no branch points and those situated in the second and the third quadrant which has branch points at the zeros of $\Gamma^{\prime}(z)$. Due to the fact that $\Gamma(z)$ is real when $z$ is real, every component has a symmetric one with respect to the real axis. Since the zeros of $\Gamma^{\prime}(z)$ are real, the branch points are located on the real axis. They form a sequence $\left(x_{n}\right)$, where $x_{0} \in(1,2)$ and for $n=1,2, \cdots$ we have that $x_{n} \in(-n,-n+1)$. Let us denote by $\alpha_{n}=\Gamma\left(x_{n}\right)$ and notice that $\alpha_{2 k}>0$ and $\alpha_{2 k+1}<0$, as seen in Figure 7(a). When $z$ belongs to the first type of component, $\lim _{\operatorname{Re} z \rightarrow+\infty} \Gamma(z)=0$ and $\lim _{\operatorname{Re} z \rightarrow-\infty} \Gamma(z)= \pm \infty$ depending on the fact that $z$ is on the pre-image of the positive, respectively negative real half axis. Similarly, if $z$ belongs to the second type of component we have that $\lim _{\operatorname{Re} z \rightarrow+\infty} \Gamma(z)= \pm \infty$ and when $z$ on the real axis tends to a pole, then the limit of $\Gamma(z)$ is $+\infty$ on the pre-image of the positive real half axis and $-\infty$ on the pre-image of the negative real half axis.

Consecutive components $\gamma_{j}^{+}$and $\gamma_{j+1}^{+}$of the first type of the pre-image of the positive real half axis bound fundamental domains $\Omega_{j}$ which are mapped conformally by $\Gamma(z)$ onto the complex plane with a slit alongside the positive real half axis. Here $j \in \mathbb{Z} \backslash\{0\}$, the real axis is between $\Omega_{-1}$ and $\Omega_{1}$ and for every $j$ the strip $\Omega_{j+1}$ is above $\Omega_{j}$. When moving on $\gamma_{j}^{-}$from $\operatorname{Re} z=-\infty$ to $\operatorname{Re} z=+\infty$ and continuing back on $\gamma_{j+1}^{+}$the corresponding domain remains to the left, therefore it is mapped by $\Gamma(z)$ onto the upper half plane. Analogously, the domain bounded by $\gamma_{j}^{+}$and $\gamma_{j}^{-}$is mapped onto the lower half plane. On 
the other hand, the involution $k(w)=-\frac{1}{\bar{w}}$ maps bijectively the upper half plane onto the lower half plane, which shows that $\Gamma_{\mid \Omega_{j}}^{-1} \circ k \circ \Gamma(z)$ is a hidden symmetry of $\Omega_{j}$.

Let us denote by $\Omega_{0}$ the domain bounded by $\gamma_{-1}^{-}, \gamma_{1}^{-}$and the first component $\delta_{1}^{+}$having a branch point. When going along $\gamma_{-1}^{-}$from $\operatorname{Re} z=-\infty$ to $\operatorname{Re} z=+\infty, \Gamma(z)$ takes all the real values from $-\infty$ to 0 and when continuing on $\gamma_{+1}^{-}$from $\operatorname{Re} z=+\infty$ to $\operatorname{Re} z=-\infty$ it will take the same values once more. When going on $\delta_{1}^{+}$from $\operatorname{Re} z=-\infty$ in the upper half plane to the branch point, $\Gamma(z)$ will take all the values from $+\infty$ to the positive value $\alpha_{0}$ where $\Gamma^{\prime}(z)$ cancels first. Continuing in the lower half plane, the same values will be taken once more. Therefore $\Omega_{0}$ is mapped conformally by $\Gamma(z)$ onto the complex plane with slits alongside real axis from $-\infty$ to 0 and from $\alpha_{0}$ to $+\infty$. The involution $\Gamma_{\mid \Omega_{0}}^{-1} \circ k \circ \Gamma(z)$ is a symmetry of this domain.

Let $\Delta_{j}^{+}$and $\Delta_{j}^{-}$be the domains bounded by $\delta_{j}$ and $\delta_{j+1}$ in the upper half plane, respectively lower half plane, $j=1,2, \cdots$. It can be easily seen that each one of them is mapped by $\Gamma(z)$ onto the complex plane with slits alongside the real axis from $-\infty$ to $\alpha_{2 j+1}$ and from $\alpha_{2 j}$ to $+\infty$ and that these domains have hidden symmetries.

Figure 7 below illustrate the graph of the real function $\Gamma(x)$ as well as the pre-image of the real axis by $\Gamma(z)$.

\section{Hidden Symmetries of Dirichlet Functions}

The knowledge we need here about Dirichlet functions can be found in [7]. We are dealing first with series of the form

$$
\zeta_{A, \Delta}(s)=\sum_{n=1}^{\infty} a_{n} \mathrm{e}^{-\lambda_{n} s}
$$

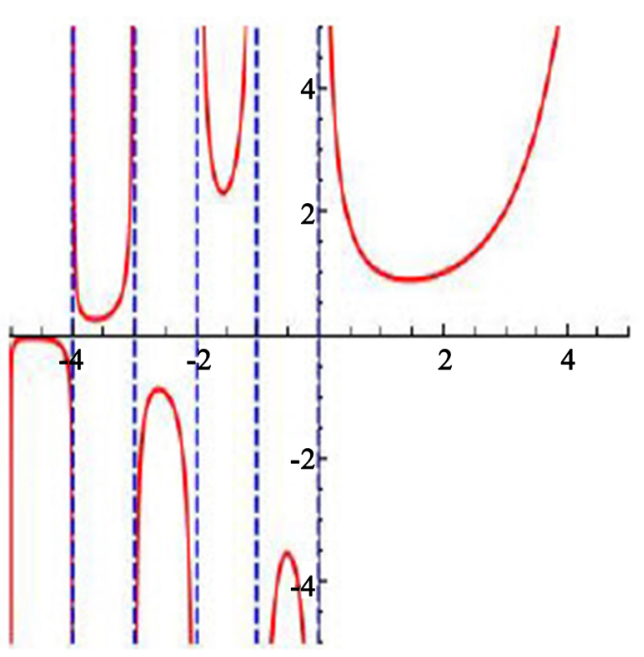

(a)

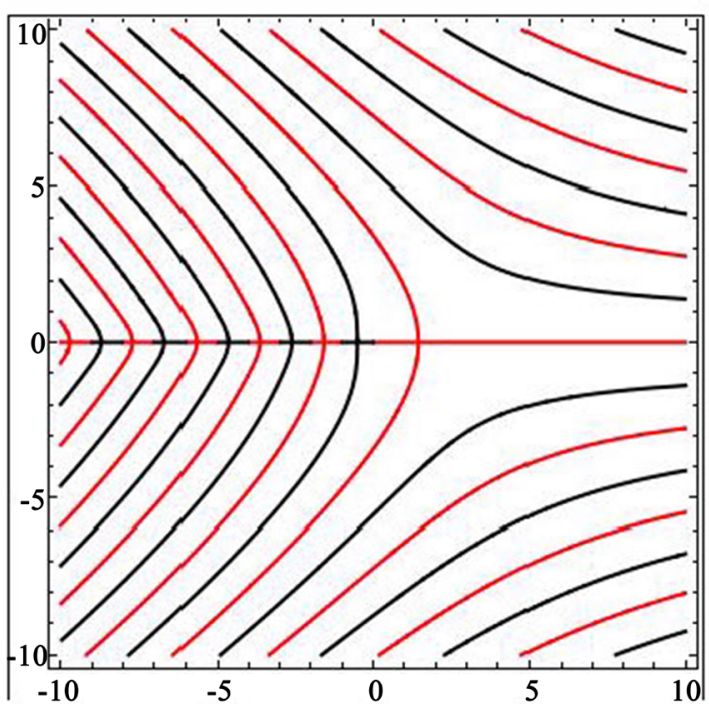

(b)

Figure 7. (a) The graphic of the real function $\Gamma(x)$; (b) The pre-image of the real axis by $\Gamma(z)$. 


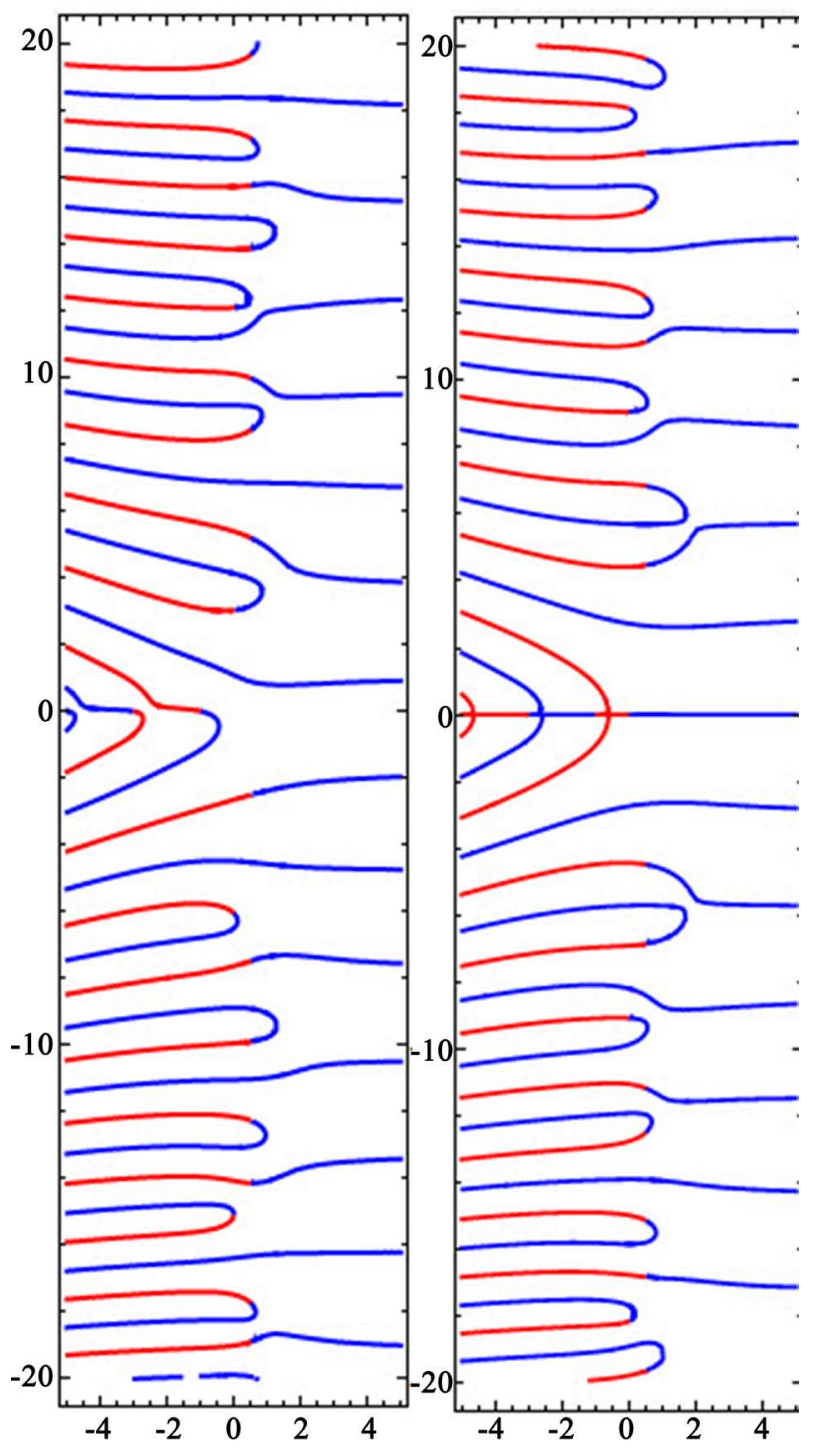

Figure 8. The pre-images of the real axis by Dirichlet L-functions defined by conplex and by real Dirichlet characters.

depending on an arbitrary sequence $A=\left\{1=a_{1}, a_{2}, \cdots\right\}$ of complex numbers, called the coefficients of (20) and on a non decreasing sequence of non negative numbers $\Lambda=\left\{0=\lambda_{1} \leq \lambda_{2} \leq \cdots\right\}$, the exponents of (20). Under certain conditions on $A$ and $\Lambda$ (see [7]) the series (20) can be continued as a meromorphic function in the whole complex plane. We keep the notation $\zeta_{A, \Delta}(s)$ for this function and we call it Dirichlet function.

It is known (see [8]) that for any Dirichlet function the complex plane admits a partition into infinitely many horizontal strips bounded by curves $\Gamma_{k}^{\prime}, k \in \mathbb{Z}$ which are mapped bijectively by $\zeta_{A, \Delta}(s)$ onto the interval $(1,+\infty)$ of the real axis. Consecutive curves $\Gamma_{k}^{\prime}$ and $\Gamma_{k+1}^{\prime}$ bound infinite strip $S_{k}$ which are mapped by $\zeta_{A, \Delta}(s)$ not necessarily injectively onto the complex plane with a slit alongside the interval $(1,+\infty)$. We denote by $S_{0}$ the strip containing the origin. It has been shown that for $k \neq 0$ every strip $S_{k}$ can be partitioned into 
finitely many sub strips whose interiors $\Omega_{k, j}$ are mapped conformally (hence bijectively) onto the complex plane with some slits. These are the fundamental domains of $\zeta_{A, \Delta}(s)$. The strip $S_{0}$ contains infinitely many fundamental domains.

When all $a_{n}$ are real the fundamental domains are symmetric two by two with respect to the real axis. Otherwise there are no obvious symmetries of Dirichlet functions. However, we can show that the fundamental domains contain hidden symmetries. Indeed, if a fundamental domain $\Omega$ is mapped conformally by $z=\zeta_{A, \Delta}(s)$ onto the complex plane $(z)$ with a slit $\delta$, let us denote by $\delta^{\prime}$ the image of $\delta$ by the involution $k(z)$ and let $\gamma$ be the image of $\delta^{\prime}$ by the conformal mapping $\zeta_{A, \Delta_{\Omega}}^{-1}$. Then $z=\zeta_{A, \Delta}(s)$ maps conformally the domain $\Delta=\Omega \backslash \gamma$ onto the complex plane with a slit alongside $\delta \bigcup \delta^{\prime}$. The antianalytic involution $\zeta_{A, \Delta_{\mid}}^{-1} \circ k \circ \zeta_{A, \Delta}(s)$ is a hidden symmetry of $\Delta$.

Figure 8 illustrates the symmetries in the case of real $a_{n}$ and lack of obvious symmetries when some $a_{n}$ are complex. However every fundamental domain contains hidden symmetries.

\section{Conflicts of Interest}

The authors declare no conflicts of interest regarding the publication of this paper.

\section{References}

[1] Ahlfors, L.V. and Sario, L. (1960) Riemann Surfaces. Princeton University Press, Princeton.

[2] Barza, I., Ghisa, D. and Ianus, S. (1998) Some Remarks on Nonorientable Surfaces, Publications de PInstitut Mathématique, 63, 47-54.

[3] Barza, I. and Ghisa, D. (1998) Explicit Formulas for Green's Functions on the Annulus and on the Möbius Strip. Acta Applicandae Mathematicae, 54, 289-302. https://doi.org/10.1023/a:1006150004533

[4] Ghisa, D. (2012) Fundamental Domains and the Riemann Hypothesis. Lambert Academic Publishing, Germany.

[5] Ahlfors, L.V. (1979) Complex Analysis. Mc-Graw-Hill, New York.

[6] Andreian-Cazacu, C. and Ghisa, D. (2011) Fundamental Domains of Gamma and Zeta Functions. IJMMS, 2011, Article ID: 985323, 21 p.

[7] Ghisa, D. (2019) Fundamental Domains of Dirichlet Functions. In: Mladenov, I.M., Pulovand, V. and Yoshioka, A., Eds., Geometry, Integrability and Quantization, Sofia, 131-160. https://doi.org/10.7546/giq-20-2019-131-160

[8] Ghisa, D. (2017) The Geometry of the Mappings by General Dirichlet Series. Advances in Pure Mathematics, 7, 1-20. 